\title{
Bright knots along spiral arms in disk galaxies
}

\author{
P. Grosbøl ${ }^{1}$ and H. Dottori ${ }^{2}$ \\ ${ }^{1}$ European Southern Observatory, Karl-Schwarzschild-Str. 2, 85748 Garching, DE \\ email: pgrosbol@eso.org \\ ${ }^{2}$ Instituto de Física, Univ. Federal do Rio Grande do Sul, Av. Bento Gonçalves 9500, \\ 91501-970 Porto Alegre, RS, BR \\ email: dottori.voy@terra.com.br
}

\begin{abstract}
Many spiral galaxies show bright knots along their arms on high resolution K-band images. Spectroscopy of such knots suggests that they are very young stellar clusters which formation was triggered by a large-scale front associated to a density wave. We have studied a sample of around 80 disk galaxies (with $i<65^{\circ}$ ) for which deep K-band maps with a resolution of $<1^{\prime \prime}$ are available and present preliminary statistics of such bright knots.
\end{abstract}

Keywords. galaxies: spiral, galaxies: star clusters, infrared: galaxies

\section{Conclusions}

Images of spiral galaxies in the near-infrared (NIR) K-band appear much smoother than those in visual bands since NIR maps are dominated by older stellar populations and are less affected by dust attenuation. Never the less, bright knots along spiral arms on K-band images are frequent (Grosbøl \& Patsis 1998). Such knots were studied by Patsis et al. (2001) using narrow band filters and by Grosbøl et al. (2006) using K-band spectroscopy. These studies suggest that the knots are very young stellar clusters which formation is triggered by a front associated to a density wave. Investigating such clusters on K-band maps has the advantage that they are easy to identify and more reliable statistics can be obtained due to the low attenuation by dust.

Deep NIR K-band maps of 83 spiral galaxies were studied and bright knots along their spiral arms were identified. A preliminary analysis found that:

- more than half of the spirals show bright knots along their arms in the K-band,

- frequency of knots in a galaxy correlates with HI mass but not with spiral type,

- knots are very well aligned along the spiral arms over a significant radial range, and

- galaxies with strong spiral perturbations in $\mathrm{K}$ also show many knots

A detailed study of such young stellar clusters may be used to constrain their star formation history and thereby improve the understanding of how density waves may trigger formation of massive stellar clusters. If accurate ages can be determined for such clusters, one may estimate the location of their formation relative to the density wave and derive parameters such as pattern speed.

\section{References}

Grosbøl, P. \& Patsis, P.A. 1998, A\&A 336, 840

Patsis, P.A., Héraudeau, P. \& Grosbøl, P. 2001, A\&A 370, 875

Grosbøl, P., Dottori, H. \& Gredel, R. 2006, A\& A 453, L25 